\title{
ESTABILIDADE TEMPORAL E CORRELATOS DESENVOLVIMENTAIS DO TRAÇO DE NEUROTICISMO EM CRIANÇAS EM FASE ESCOLAR ${ }^{1}$
}

\author{
Marcela Mansur-Alves ${ }^{*}$ \\ Carmen Flores-Mendoza
}

\begin{abstract}
RESUMO. Neuroticismo (N) é um dos traços de personalidade mais amplamente estudados na psicologia da personalidade. As evidências atuais apontam a importância de se estudar o impacto do neuroticismo em vários aspectos do desenvolvimento infantil. Assim sendo, o presente estudo objetivou primeiramente verificar a consistência temporal de $\mathrm{N}$ e suas relações com determinados aspectos do desenvolvimento infantil. Participaram do estudo 368 crianças de uma escola pública federal da cidade de Belo Horizonte, às quais foram aplicados testes de inteligência e de desempenho escolar e dois instrumentos de autorrelato da personalidade, bem como foi realizada uma avaliação antropométrica. Ademais, os pais das crianças preencheram um questionário de funcionamento adaptativo. Os resultados apontam a existência de moderada estabilidade temporal de $\mathrm{N}$. Não foram encontradas associações significativas entre $\mathrm{N}$ e as medidas antropométricas. Não obstante, o neuroticismo foi capaz de predizer o desempenho escolar, mesmo quando se controlou o efeito da inteligência, e se associa também ao funcionamento adaptativo das crianças. Conclui-se que a dimensão neuroticismo possui significativo impacto no desenvolvimento das crianças em fase escolar.
\end{abstract}

Palavras-chave: Neuroticismo; consistência temporal; desenvolvimento infantil.

\section{STABILITY AND DEVELOPMENTAL CORRELATES OF NEUROTICISM TRAIT IN SCHOOL CHILDREN}

\begin{abstract}
Neuroticism (N) is one of the most studied traits in the history of the personality psychology. The current evidence has pointed the importance of studying the impact of Neuroticism in some aspects of child development. Therefore, this study aimed to verify the temporal stability of $\mathrm{N}$ and its relations with some aspects of child development. The data were collected in a group of 368 children from a federal public school of Belo Horizonte. To all participants of the study were applied completed intelligence and school performance tests, two instruments of self-report of personality and the anthropometric assessment. Children's parents filled out a questionnaire of adaptive functioning. The results point to the existence of moderated temporal stability of $\mathrm{N}$ in both self-reports used. There were no significant associations between $\mathrm{N}$ and anthropometric measures. The Neuroticism predicted the academic performance, even when the effect of intelligence was controlled. $\mathrm{N}$ also had association with the adaptive functioning of children. It was concluded that Neuroticism, in fact, has significant impact on the development of children in school years.
\end{abstract}

Key words: Neuroticism; temporal stability; child development.

\section{ESTABILIDAD TEMPORAL Y CORRELATOS DE DESARROLLO DEL RASGO NEUROTICISMO EN NIÑOS ESCOLARES}

RESUMEN. Neuroticismo (N) es uno de los rasgos de personalidad más amplamente estudiados en la psicología de la personalidad. Las evidencias actuales muestran la importancia de estudiar el impacto del neuroticismo en varios aspectos del desarrollo infantil. Por tal razón, el presente estudio objetivó primeramente verificar la consistencia temporal de $\mathrm{N}$ y sus relaciones con determinados aspectos del desarrollo infantil. Han participado del estudio 368 niños de una escuela pública federal de la ciudad de Belo Horizonte, a los cuales fueron administrados testes de inteligencia y de desempeño escolar asi

1 Apoio: Fundação de Amparo à Pesquisa do Estado de Minas Gerais-FAPEMIG.

* Psicóloga e Doutoranda do Programa de Pós-Graduação em Neurociênciada Universidade Federal de Minas Gerais-UFMG. Membro do Laboratório de Avaliação das Diferenças Individuais.

\# Professora Adjunta do Departamento de Psicologia e do Programa de Pós-Graduação em Neurociência da UFMG. Coordenadora do Laboratório de Avaliação das Diferenças Individuais. 
como dos instrumentos de autoinforme de la personalidad y una evaluación antropométrica. Finalmente, los padres de los niños respondieron a un cuestionario sobre el funcionamiento adaptativo. Los resultados indicaron la existencia de una moderada estabilidad temporal de N. No se encontraron asociaciones significativas entre $\mathrm{N}$ y las medidas antropométricas. No obstante, el neuroticismo fue capaz de predecir el desempeño escolar, mismo después de controlar el efecto de la inteligencia, asociandose también al funcionamiento adaptativo de los niños. Se concluye que la dimensión neuroticismo posee significativo impacto en el desarrollo de los niños escolares.

Palabras clave: Neuroticismo; consistencia temporal; desarrollo infantil.

Dentre as dimensões da personalidade postuladas pelo modelo dos "Cinco Grandes Fatores", o neuroticismo pode ser considerado uma das mais amplamente estudadas no campo da psicologia da personalidade (Caspi, Roberts \& Shiner, 2005). Segundo De Raad (2000), o neurrrroticismo está presente em 5.222 das 17.262 referências sobre as cinco grandes dimensões da personalidade registradas em abstracts desde 1887. Já em 1968, Wiggins ressaltava o consenso no que diz respeito à extroversão e ao neuroticismo como os "Dois Grandes" fatores da personalidade (De Raad, 2000). Matthews, Deary e Whiteman (2003) afirmam que a susceptibilidade às emoções negativas e a intensidade da reação emocional (Neuroticismo) podem ser identificadas em diferentes períodos dos estudos da personalidade humana, muito embora os nomes dados a essa dimensão possam variar de acordo com a época e o modelo teórico utilizado. De acordo com os esquemas humorais do temperamento proposto por Wilhelm Wundt, o neuroticismo seria representado pelo que o autor chamou de "emoções fortes" (intensas). No modelo temperamental de Mary Rothbart e John Bates (Shiner, 1998), o neuroticismo é chamado de emocionalidade negativa. O termo emocionalidade negativa também é utilizado no modelo dos Três Fatores de Auke Tellegen e no questionário que o operacionaliza (Multidimensional Personality Questionnaire(MPQ). De acordo com Matthews et al. (2003), o fator neuroticismo não só é representado em diferentes modelos e em diversos questionários de personalidade, mas também há uma elevada correspondência entre eles.

O neuroticismo relaciona-se, entre outras coisas, à reatividade emocional do indivíduo, às tendências para preocupação, à susceptibilidade ao humor negativo e à propensão à psicopatologia. De acordo com Eysenck (1959), o neuroticismo é o fator da personalidade que pode ser mensurado com a mesma validade e confiabilidade que a inteligência. Por exemplo, Terracciano, Costa e McCrae (2006) demonstram alto grau de estabilidade para o neuroticismo $(r=0,78)$ para as idades entre 30 e 69 anos. As tendências neuróticas foram pela primeira vez medidas por meio de um inventário durante a Primeira Guerra Mundial. O Woodworth's Personal Data Sheet, nome pelo qual ficou conhecido o inventário, objetivava avaliar as habilidades dos soldados em lidar com situações de estresse (De Raad, 2000). Nos anos seguintes a Primeira Grande Guerra, outros inventários foram desenvolvidos com o objetivo de mensurar algumas características do neuroticismo, como a hipersensibilidade, a depressão e a imaturidade emocional, em universitários, militares e na população geral (De Raad, 2000). A partir de então, todas as taxonomias do temperamento e da personalidade incluem um traço relacionado à tendência a experienciar o mundo como algo estressante e ameaçador (Caspi et al., 2005).

No que se refere às taxonomias do temperamento e da personalidade infantil, os termos mais comumente utilizados são: neuroticismo, emocionalidade negativa ou afetividade negativa (Shiner \& Caspi, 2003), sendo que os dois últimos acentuam as tendências a vivenciar emoções negativas como características nucleares do referido traço. Segundo Rothbart e Bates (1998), nas crianças, a emocionalidade negativa envolve dois agrupamentos relacionados, embora distintos, de emoções negativas: o anxious distress (aflição ansiosa ou somente ansiedade) e o irritable distress (aflição irritável ou somente irritabilidade). $\mathrm{O}$ anxious distress está relacionado a emoções negativas, como culpa, medo, insegurança e ansiedade, que são internalizantes. De acordo com Shiner e Caspi (2003), tal tendência a experimentar emoções negativas internalizantes tem sido identificada como um fator de vulnerabilidade a vários tipos de psicopatologias adultas. Em contraste, o irritable distress envolve emoções negativas, como irritabilidade, raiva e frustração, que são externalizantes. Essas emoções hostis são frequentemente evocadas por limitações ou barreiras externas colocadas pelos adultos aos comportamentos ou vontades da criança (Caspi et al., 2005). Esses dois agrupamentos de emoções negativas parecem seguir diferentes caminhos desenvolvimentais até atingir a configuração estrutural da dimensão de neuroticismo observada em adultos. 
O neuroticismo é uma dimensão sistematicamente pesquisada em estudos da genética comportamental (delineamento de gêmeos, de adoção e genética molecular), em estudos psicobiológicos, neuropsicológicos, da psicologia cognitiva e em estudos que buscam estabelecer o alcance de tais dimensões como preditoras de eventos importantes de vida (para maiores detalhes ver Matthews et al., 2003). $\mathrm{O}$ construto neuroticismo é relevante em uma variedade de contextos, entre os quais se podem citar os contextos clínicos, de aprendizagem e educação, na predição de problemas relacionados à saúde, satisfação na vida e funcionamento adaptativo (Caspi et al., 2005; De Raad, 2000; Laidra, Pullmann \& Allik, 2007; Nederkoorn, Braet, Van Eijs, Tanghe \& Jansen, 2006; Rubinstein, 2006; Shiner \& Caspi, 2003).

Neste sentido, o presente estudo objetivou verificar a estabilidade temporal e potencial preditivo da dimensão de neuroticismo em aspectos importantes da capacidade física e psicológica de crianças escolares. Visou-se verificar se e como o neuroticismo interfere na capacidade adaptativa de crianças em fase escolar.

\section{MÉTODO}

\section{Amostra}

Participaram do estudo 368 sujeitos, matriculados em turmas da $3^{\mathrm{a}}$ à $8^{\mathrm{a}}$ série de uma escola pública federal da cidade de Belo Horizonte, sendo, em média, 60 alunos de cada série. Essa escola foi escolhida pelo fato de nela entrarem alunos diferentes origens socioeconômicas, por meio de sorteio, o que a torna naturalmente uma amostra aleatorizada da população da faixa etária compreendida pelo estudo. A opção por essas séries se deu pela necessidade de domínio de leitura por parte das crianças, tendo-se em vista que delas seria solicitado o preenchimento de questionários de autorrelato da personalidade. $\mathrm{O}$ sexo masculino representou 52,2\% $(\mathrm{N}=192)$ da amostra. A idade média da amostra total foi de 11,46 anos com desvio padrão de 1,78 .

\section{Instrumentos}

No estudo foram utilizados os instrumentos a seguir numerados e descritos.

1- Big Five Questionnaire for Children -BFQ-C - versão de autoavaliação (Barbaranelli, Caprara \& Rabasca, 1998): é um instrumento que visa mensurar as cinco dimensões da personalidade propostas pelo modelo dos cinco grandes fatores (extroversão, neuroticismo, cordialidade, responsabilidade e abertura mental) em crianças e adolescentes. A versão que está em processo de adaptação para o contexto brasileiro pelo Laboratório de Avaliação das Diferenças Individuais (LADI) e que foi utilizada no presente estudo consiste em uma versão única para crianças de 9 a 15 anos $^{2}$. Essa única versão consta de 75 itens, sendo 65 da versão original e 10 itens criados como alternativos aos itens que, em estudos anteriores conduzidos pelo laboratório, mostraram menor correlação com o fator correspondente. As respostas são dadas em uma escala do tipo Likert de cinco pontos para todas as faixas etárias (nunca/ pouco/ às vezes sim às vezes não/ bastante/ sempre). Os índices de consistência interna para todos os fatores ficaram dentro do limite aceitável, variando de 0,743 para extroversão a 0,859 para responsabilidade (Mansur-Alves, 2007).

2 - Eysenck Personality Questionnaire Junior - EPQ-J (Eysenck \& Eysenck 1975/1998): é uma medida de autoavaliação das diferenças individuais nas três dimensões de personalidade (Neuroticismo, Extroversão, Psicoticismo) do modelo de Hans Eysenck, em crianças e adolescentes de 8 a 15 anos de idade. O instrumento consta, ainda, de uma escala de validade (sinceridade), cujo objetivo é avaliar a compreensão dos itens pelo respondente, bem como sua honestidade ao responder ao teste. Deve-se registrar que a escala de sinceridade constitui apenas uma estratégia de verificação da validade das respostas. $O$ instrumento está sendo adaptado também pelo LADI. As quatro escalas perfazem um total de 81 itens, com formato dicotômico de resposta (Sim ou Não). Os índices de consistência interna foram 0,65 para extroversão; 0,78 para neuroticismo; 0,72 para psicoticismo e 0,79 para sinceridade (Mansur-Alves, 2007).

3- Matrizes Progressivas de Raven - Escala Geral (CEPA, 2001): o teste de Raven é uma medida de inteligência não verbal que avalia mais especificamente o fator "g", proposto por Spearman, de um modo que fosse reduzida a um mínimo a contaminação pela variação do conhecimento daqueles que fossem testados. A Escala Geral foi utilizada para todas as faixas etárias, em prefência à Escala Colorida, para capturar a variabilidade desde os nove aos quize anos de idade com um mesmo instrumento.

4- Teste de Desempenho Escolar-TDE (Stein, 1994): foi elaborado por Stein (1994) e através dele

2 A versão original em italiano consta de duas versões: uma para as idades entre 8 e 10 anos e outra para as idades entre 11 e 14 anos. A única diferença entre elas é a escala de resposta. 
avaliam-se de forma objetiva as capacidades em leitura, escrita e aritmética, consideradas fundamentais para um bom desempenho escolar. $\mathrm{O}$ instrumento é composto por três subtestes: o de escrita, o de aritmética e o de leitura.

5 - Questionário de Funcionamento Adaptativo: foi elaborado um questionário a ser preenchido pelos pais, contendo seis itens que objetivavam fazer uma breve triagem de alguns aspectos do funcionamento adaptativo da criança em seu ambiente doméstico. Os itens avaliavam relacionamento interpessoal, dificuldades relacionadas ao sono e à alimentação, estimulação e sintomas psicológicos e físicos da ansiedade. Os itens foram elaborados tendo como base o Child Behavior Checklist /CBCL (Achenbach, 1991). A resposta é era dada com base numa escala do tipo Likert de três pontos (Sim/ Não/ Às vezes).

6- Medidas antropométricas para avaliação de peso e altura: o peso foi medido usando uma balança digital com capacidade de até $150 \mathrm{~kg}$, que zera automaticamente após a retirada da carga. A altura foi medida em posição ereta, sem sapatos e com uma fita métrica flexível acoplada à parede da sala.

\section{Procedimentos}

Entre os meses de março e maio de 2006 os alunos foram avaliados por meio do teste Raven, TDE, do BFQ-C, do EPQ-J e das variáveis antropométricas (peso e altura). Com exceção do TDE e da avaliação antropométrica, todas as avaliações se deram de forma coletiva em local previamente cedido pela escola. A avaliação foi feita com o auxílio de uma equipe de avaliadores composta de alunos de graduação em Psicologia e treinada especialmente para o projeto. Em outubro de 2006, seis meses após a primeira aplicação, as crianças da $3^{\mathrm{a}}, 4^{\mathrm{a}}$ e $5^{\mathrm{a}}$ séries e os adolescentes da $6^{\mathrm{a}}, 7^{\mathrm{a}}$ e $8^{\mathrm{a}}$ séries foram novamente avaliados com o BFQ-C e com o EPQ-J. As avaliações ocorreram de forma coletiva nas dependências da própria escola.

Simultaneamente ao reteste dos questionários de personalidade foi solicitado aos pais que respondessem ao questionário de funcionamento adaptativo. O chefe da Seção de Ensino da escola se encarregou de enviar os questionários aos pais e de recolhê-los. Como o questionário foi enviado juntamente com os documentos de rematrícula do aluno na escola, somente os pais dos alunos da $8^{\mathrm{a}}$ série não responderam a ele.

\section{RESULTADOS}

As análises apresentadas a seguir foram realizadas tendo como base somente aqueles sujeitos a respeito dos quais havia dados completos de ambas as escalas de autorrelato da personalidade consideradas no estudo. Destarte fora analisados os dados de 368 alunos, distribuídos entre a $3^{\mathrm{a}}$ e a $8^{\mathrm{a}}$ série do Ensino Fundamental, havendo distribuição uniforme dos alunos por série. Em primeiro lugar, verificou-se a ocorrência de uma distribuição normal dos resultados para efeito de aplicação de técnicas paramétricas. A distribuição normal aceita é aquela em que a largura (Skewness) e a altura (Kurtosis) não são superiores em 2,5 vezes em relação a seu erro padrão. Para tanto se divide a estatística pelo erro padrão. Na tabela 1 apresentam-se as descritivas (valores mínimo e máximo, média, desvio padrão, Skewness e Kurtosis) dos resultados obtidos em neuroticismo para cada uma das escalas de personalidade.

Tabela 1. Média, Desvio-padrão, Skewness e Kurtosis de N Para as Duas Escalas de Personalidade Consideradas

\begin{tabular}{lccccccccccccc}
\hline Neuroticismo & \multicolumn{4}{c}{ Descritivas } & \multicolumn{4}{c}{ Skewness/Valor z Kurtosis/Valor z } & \multicolumn{2}{c}{$\begin{array}{c}\text { Diferença [curva teórica vs. curva } \\
\text { observada] }\end{array}$} \\
\cline { 2 - 12 } & N & Min & Max & $\underline{\text { M }}$ & $\underline{\text { DP }}$ & Estatística & EP & Estatística & EP & Skewness & Kurtosis \\
\hline BFQ-C & 368 & 19 & 61 & 35,56 & 7,978 & 0,008 & 0,127 & $-0,122$ & 0,254 & 0,062 & $-0,480$ \\
EPQ-J & 368 & 14 & 28 & 20,12 & 3,383 & 0,032 & 0,127 & $-0,266$ & 0,254 & 0,251 & $-1,047$ \\
\hline
\end{tabular}

Nota: EP = erro padrão

Conforme pode ser observado na tabela, os valores são menores que 2,5, tanto para os resultados do BFQ-C quanto para os do EPQ-J, o que permite aceitar a distribuição normal dos escores.

\section{Associação do Neuroticismo com variáveis biológicas}

O coeficiente de correlação de Pearson foi utilizado para verificar o grau de associação entre o neuroticismo e a inteligência (medida pelo RavenEscala Geral) e o índice de massa corporal (IMC). A inteligência foi aqui considerada como uma variável biológica, haja vista as inúmeras constatações de que a maior parte das diferenças individuais em inteligência (medida por qualquer teste, verbal ou não verbal) pode ser explicada por variações genéticas (Kovacs \& Plomin, 2006). O teste Raven foi escolhido porque, 
quando comparado a outras medidas de habilidades cognitivas, apresenta uma das maiores saturações na inteligência psicométrica, chamada também de fator $g$ (Jensen, 1998).

Sendo assim, os valores brutos de inteligência foram transformados em escores $\mathrm{z}$, controlando-se a variável idade, dado o efeito desenvolvimental esperado. O IMC é uma medida estatística do peso de um indivíduo pela sua altura, sendo considerado um bom indicador da quantidade de gordura corporal existente (World Health Organization-WHO, 2006). O IMC pode ser calculado utilizando-se a seguinte fórmula:

$$
I M C=\operatorname{Peso}(K g) / \text { Altura }^{2}(m)
$$

Para crianças e adolescentes, o valor do IMC, calculado com base na fórmula acima, é transformado em postos percentílicos, para os quais se consideram a idade e o sexo da criança ou do adolescente. A interpretação do IMC com base em percentis é utilizada por duas razões principais (Centers for Disease Control and PreventionCDC, 2007): 1) a quantidade de gordura no corpo muda até os 20 anos, em decorrência de processos desenvolvimentais normais; 2) a quantidade de gordura corporal é diferente para meninos e meninas.

Dessa forma, para a presente análise, o IMC bruto foi transformado em escores tipificados, controlando-se a idade e o sexo da criança, haja vista os fatores mencionados acima. A amostra considerada para essa análise foi de 356 crianças avaliadas com o Raven Escala Geral e as variáveis antropométricas (peso e altura).

Os resultados encontrados mostram que não há correlações significativas entre o $\mathrm{N}$ - tanto medido pelo BFQ-C quanto pelo EPQ-J - e a inteligência. Correlações significativas e positivas foram encontradas entre o $\mathrm{N}$ do BFQ-C e o IMC $(\underline{r}=0,151 ; \underline{p}<0,05)$. Também foi encontrada correlação significativa entre a inteligência e o IMC ( $\underline{r}=-0,111 ; \underline{p}<0,05)$. A associação negativa, neste caso, indica que crianças mais inteligentes tendem a apresentar menor IMC. Quando se controla o efeito da inteligência, a associação entre o $\mathrm{N}$ e o IMC diminui $(\underline{\mathrm{r}}=$ $0,140 ; \underline{p}<0,05)$. De forma semelhante, quando se controla o $\mathrm{N}$, a associação entre inteligência e IMC se reduz $(\mathrm{r}=-0,105 ; \underline{\mathrm{p}}<0,05)$. Esses resultados parecem indicar que a contribuição do neuroticismo e da inteligência nas diferenças individuais em IMC é independente.

\section{Associação de $\mathbf{N}$ com desempenho escolar e inteligência}

O primeiro passo para verificar a associação entre $\mathrm{N}$ e desempenho escolar foi certificar-se da existência de relação entre esse último e a inteligência, dado que a literatura das diferenças individuais é farta em apresentar informações sobre o alto poder de predição da inteligência no desempenho escolar (Jensen, 1998). Para tanto, realizou-se uma correlação de Pearson entre os escores tipificados do Raven (medida de inteligência) e os escores tipificados do TDE, controlando-se a idade, haja vista o efeito desenvolvimental esperado. A amostra compreendeu 250 crianças avaliadas com o Teste de Desempenho Escolar.

A correlação encontrada entre o Raven e o TDE foi de $0,376(\underline{p}<0,01)$, indicando que quanto maior a inteligência da criança, melhor será o seu rendimento na escola; portanto, a inteligência foi controlada nas análises. Não foram encontradas correlações significativas entre o $\mathrm{N}$ medido pelo BFQ-C e as pontuações no $\operatorname{TDE}(\underline{\mathrm{r}}=-0,086 ; \underline{\mathrm{p}}=0,184)$; entretanto, correlações negativas e significativas, embora baixas, foram encontradas entre o $\mathrm{N}$, medido pelo EPQ-J, e o TDE ( $\underline{r}=-0,186 ; \underline{p}=0,004)$, mesmo controlando-se a inteligência. Esse resultado significa que com a elevação das pontuações em $\mathrm{N}$ pode ser observada uma queda no desempenho escolar.

Com o intuito de explorar de forma mais consistente a inter-relação existente entre o conjunto de variáveis acima, realizou-se uma análise de regressão linear. Os resultados indicam que as duas variáveis que ingressaram como independentes explicaram $17,1 \%$ da variância em $\operatorname{TDE}\left(\underline{\mathrm{R}}^{2}=0,171\right)$. Tal modelo foi estatisticamente significativo $(\underline{F}(2,237)=24,46 ; \underline{p}<0,001)$. Na Tabela 2 se apresenta a contribuição de cada uma das variáveis para a equação de regressão.

Tabela 2. Variáveis Preditoras do TDE: Inteligência e Neuroticismo (EPQ-J)

\begin{tabular}{lccc}
\hline \multirow{2}{*}{ Modelo } & \multicolumn{2}{c}{$\begin{array}{c}\text { Coeficientes Não } \\
\text { padronizados }\end{array}$} & $\begin{array}{c}\text { Coeficientes } \\
\text { Padronizados }\end{array}$ \\
\cline { 2 - 4 } & $\underline{\mathbf{B}}$ & Erro Padrão & $\boldsymbol{\beta}$ \\
\hline Inteligência & 0,380 & 0,59 & $0,379^{* *}$ \\
Neuroticismo & $-0,166$ & 0,57 & $-0,172^{* *}$ \\
\hline
\end{tabular}

$* * \underline{\mathrm{R}^{2}}=0,171(\underline{\mathrm{N}}=240 ; \underline{\mathrm{p}}<0,01)$

Observa-se na tabela 2 que, conforme esperado, a inteligência $(\beta=0,379)$ é o atributo pessoal que mais contribui para a predição do desempenho escolar, quando comparada ao neuroticismo $(\beta=-0,172)$.

\section{Estabilidade temporal do neuroticismo}

Com a finalidade de verificar o grau de estabilidade temporal da dimensão de neuroticismo, uma subamostra de 157 crianças foi submetida a um reteste seis meses 
após a primeira avaliação. Das 157 crianças avaliadas no reteste, 79 fizeram o BFQ-C e 78 o EPQ-J. Os coeficientes de correlação teste-reteste encontrados para ambos os instrumentos são moderados e significativos [(r $=0,553 ; \mathrm{p}<0,01)$ para o BFQ-C, e $(\mathrm{r}=0,638 ; \mathrm{p}<0,01)$ para o EPQ-J]. Tais resultados evidenciam considerável estabilidade temporal da dimensão neuroticismo num intervalo de seis meses.

\section{Avaliação do Funcionamento Adaptativo}

Para essa análise foram selecionadas apenas as crianças de $3^{\mathrm{a}}$ a $5^{\mathrm{a}}$ series que obtiveram altas e baixas pontuações na escala conjunta de neuroticismo formada pelos itens do BFQ-C e EPQ-J. Para tal, os itens foram somados e transformados em escore $\mathrm{z}$ e logo em quartil. Obtiveram-se 50 crianças do quartil 1 (baixo N) e 42 crianças do quartil 4 (alto N). Das 92 crianças selecionadas, apenas 76,4\% (68) dos pais responderam ao questionário de funcionamento adaptativo. Assim sendo, as resultados descritos a seguir estão baseados numa amostra de 68 crianças.

Para verificar a existência de associação entre o N (BFQ-C + EPQ-J), avaliado pelo autorrelato, e o funcionamento adaptativo da criança, avaliado pelos pais, realizou-se uma análise de correlação de Spearman, que é um indicador não paramétrico da associação de variáveis usualmente ordinais, sendo também utilizado em amostras pequenas. Observou-se a existência de associação negativa e significativa entre o $\mathrm{N}$ avaliado pelo autorrelato e o índice de funcionamento adaptativo da criança em seu ambiente domiciliar $(\rho=-0,320 ; p$ $<0,01)$. Esse resultado indica que com a elevação do neuroticismo observa-se uma diminuição do funcionamento adaptativo da criança (ajustamento), pelo menos no que se refere ao ambiente doméstico. Com a finalidade de verificar a existência de diferenças no funcionamento adaptativo da criança associada à sua classificação (quartil) em N, realizou-se a prova de MannWhitney para amostras independentes, haja vista que a variável dependente (questionário de funcionamento adaptativo) não possui normalidade e a amostra é pequena. $\mathrm{O}$ resultado da prova de Mann-Whitney aponta a existência de diferenças significativas no funcionamento adaptativo para os dois extremos de $\mathrm{N}$ ( $\underline{\mathrm{U}}$ $=298,50 ; \underline{p}<0,01)$. Isso significa que as crianças com pontuações mais baixas em $\mathrm{N}$ possuem melhor funcionamento adaptativo do que aquelas com alto $\mathrm{N}$.

\section{DISCUSSÃO}

Nos últimos anos, um grande número de evidências tem sido compilado acerca da universalidade da dimensão neuroticismo nos vários sistemas psicobiológicos e psicométricos da personalidade humana (Matthews et al., 2003). A presente investigação objetivou verificar a validade preditiva do neuroticismo em vários aspectos do funcionamento diário de crianças em fase escolar, bem como sua consistência temporal.

Em primeiro lugar, buscou-se verificar a existência de associação entre o Neuroticismo, a inteligência e o IMC. Os resultados encontrados mostraram não haver correlações significativas entre o $\mathrm{N}$ e a inteligência; por outro lado, a associação positiva entre o $\mathrm{N}$ medido pelo BFQ-C e o IMC desaparece quando se controla a inteligência, uma vez que esta apresenta associação negativa e significativa com o IMC.

Quanto à associação entre inteligência e neuroticismo, tradicionalmente, a psicologia diferencial distingue personalidade e habilidade em termos de seus processos subjacentes (ChamorroPremuzic, Furnham \& Petrides, 2006). Dessa forma, quando se encontram correlações empíricas entre traços de personalidade e testes de QI, geralmente isto se deve a "efeitos estilísticos". Por exemplo, as associações entre a extroversão e o QI são explicadas em termos da elevada velocidade de resposta e da assertividade dos indivíduos extrovertidos, consideradas como vantagens em muitos testes psicométricos de QI (Chamorro-Premuzic \& Furnham, 2004). De maneira semelhante, Chamorro et al. (2006) demonstram que indivíduos com alto neuroticismo se saem pior em testes de habilidade verbal do que aqueles com baixo neuroticismo, especialmente em decorrência da falta de confiança dos primeiros.

No que se refere ao IMC, o neuroticismo é frequentemente associado a transtornos nutricionais, especialmente sobrepeso e obesidade. Rubinstein (2006) constatou que mulheres com sobrepeso possuem Ns mais elevados do que mulheres com peso normal. Esse autor relata, ainda, que quanto maior o $\mathrm{N}$, mais difícil se torna para as mulheres seguir uma dieta. Faith, Flint, Faiburn, Goodwin e Allison (2001) encontraram que, entre mulheres, o aumento do IMC está significativamente associado ao aumento do neuroticismo e redução da extroversão. Nederkoorn et al. (2006) afirmam ser a impulsividade, característica do neuroticismo, a principal determinante do desenvolvimento e manutenção da obesidade infantil. Csabi, Tenyi e Molnar (2000) encontraram maior proporção de sintomas depressivos em crianças obesas quando comparadas com as de peso normal,; entretanto, não se encontraram estudos de crianças com peso superior controlando-se a variável 
inteligência. $O$ presente estudo mostra que a associação positiva entre N (BFQ-C) e o IMC diminui quando se controla o efeito da inteligência.

Era de esperar, diante desses resultados, a associação negativa entre o IMC e a inteligência encontrada no presente estudo. Tal associação indica que quanto mais inteligente for a criança menor deverá ser seu IMC, portanto, também menor probabilidade de desenvolver desordens alimentares no futuro. Cournot et al. (2006) encontraram resultado semelhante num estudo desenvolvido com adultos franceses. Esses autores constataram associação negativa entre o IMC e uma bateria de testes cognitivos. Batty, Deary, Schoon e Gale (2007), em estudo longitudinal realizado com mais de 17.000 sujeitos ingleses, apontam que crianças de maior capacidade intelectual consomem mais frequentemente frutas, vegetais, carne branca e peixe, e menos quantidade de frituras e gorduras quando adultos, bem como praticam mais exercícios físicos. Tal efeito ainda não pode ser observado na amostra utilizada.

Uma das grandes preocupações no campo da Educação é buscar os determinantes do desempenho escolar, uma vez que o sucesso na escola possui papel impactante nas futuras oportunidades oferecidas ao indivíduo. Tem sido identificado um grande número de variáveis relacionadas ao sucesso e fracasso na escola, sendo a inteligência e a personalidade as mais importantes. Os índices encontrados no presente estudo são condizentes com aqueles descritos na literatura especializada, na qual a inteligência se correlaciona positivamente com o desempenho escolar, assim como o neuroticismo se relaciona de forma negativa (Laidra et al., 2007; Neisser et al. 1996). Entre os dois construtos, a regressão linear mostrou que a inteligência é o melhor preditor do desempenho escolar. Esse resultado é coerente com os demais descritos na área.

Diversas investigações encontraram resultados semelhantes acerca da associação entre $\mathrm{N}$ e desempenho escolar (Laidra et al., 2007; Pacheco, 2003). Chamorro-Premuzic e Furnham (2004) e Chamorro-Premuzic et al. (2006) argumentam que a associação negativa existente entre o neuroticismo e o desempenho escolar se deve ao fato de indivíduos altamente neuróticos tenderem a experimentar grande ansiedade em situações de testagem, o que prejudica o desempenho de tais indivíduos em exames e testes escolares. Chamorro-Premuzic, Furnham, Dissou e Heaven (2005) acreditam, ainda, que o desempenho dos indivíduos em exames escolares pode estar mediado pela relação existente entre as características de personalidade e o tipo de avaliação utilizado em sala de aula. Em específico, eles apontam que indivíduos com elevado neuroticismo se saem pior em exames orais ou em avaliações contínuas. Um dado interessante acerca da relação entre $\mathrm{N}$ e desempenho escolar diz respeito ao baixo coeficiente de correlação na presente investigação quando comparado ao encontrado em investigações internacionais. A disparidade entre os coeficientes encontrados pode estar relacionada à mediação de tal relação pela ansiedade. A ansiedade das crianças da presente amostra ante a realização de provas de desempenho acadêmico pode não ser tão alta, quando se leva em consideração que elas já estão acostumadas a esse tipo de prova. A escola participante da pesquisa se caracteriza pela constante presença de pesquisas científicas em seu ambiente, incluindo a pesquisa da qual a presente amostra faz parte.

Com relação à existência de associação entre o desempenho escolar e o $\mathrm{N}$ medido pelo EPQ-J e a inexistência de tal relação para o BFQ-C, poder-se-ia dizer que ela estaria relacionada à diferença de conteúdos entre os dois instrumentos. Parece, pois, que a conceituação teórica de $\mathrm{N}$ do EPQ-J permite melhor capturar a variabilidade do desempenho escolar em função de $\mathrm{N}$. O fato de a conceituação teórica do EPQ-J ser adequada para capturar a variabilidade no desempenho escolar associado a $\mathrm{N}$ é confirmado pela pesquisa realizada por Pacheco (2003) entre escolares brasileiros de 6 a 10 anos de idade. A autora utilizou um questionário de avaliação da personalidade baseado no modelo de Eysenck e também encontrou associação negativa entre $\mathrm{N}$ e desempenho escolar. Pesquisas nacionais utilizando o BFQ-C são praticamente inexistentes.

A presente investigação objetivou, ainda, estudar a estabilidade do neuroticismo na infância e adolescência. Para tanto, o BFQ-C e o EPQ-J foram aplicados a uma subamostra de crianças em duas ocasiões diferentes, com um intervalo de seis meses entre uma e a outra. Os coeficientes de correlação teste-reteste encontrados aqui são de magnitude similar àqueles encontrados na meta-análise realizada por Roberts e DelVecchio (2000) com mais de 152 estudos. Esses autores apontam que a correlação média entre duas avaliações de personalidade é de 0,40 para a infância e adolescência. Especificamente com relação ao $\mathrm{N}$, os autores encontram um coeficiente de estabilidade diferencial de 0,54. Em geral, os coeficientes de estabilidade diferencial, relatados na literatura da área e encontrados aqui, são mais baixos para crianças e adolescentes, aumentando progressivamente à medida que se avança no ciclo 
vital (Roberts \& DelVecchio, 2000). Essa diferença entre faixas etárias na magnitude dos coeficientes de estabilidade se deve ao fato de que, segundo Terracciano et al. (2006), a personalidade humana está sujeita a mais mudanças até a idade de 30 anos. A partir dessa idade, embora as características de personalidade ainda possam sofrer influências ambientais e constitucionais, a mudança observada é muito pequena, para a maior parte das pessoas. Já a personalidade da criança, apesar de estar mais suscetível à influência ambiental, ainda mostra coeficientes razoáveis de estabilidade diferencial, o que poderia indicar possíveis origens constitucionais.

Uma das informações mais importantes é a forma como a criança experiencia, interpreta e responde ao mundo à sua volta (Shiner \& Caspi, 2003). A personalidade da criança pode torná-la vulnerável ou protegê-la de resultados desenvolvimentais maladaptativos. Assim sendo, o presente estudo encontrou correlações negativas entre o $\mathrm{N}$ e o funcionamento adaptativo, indicando que, quanto maior $\mathrm{o}$ neuroticismo da, criança maiores são também as dificuldades interpessoais, alimentares e os sintomas físicos e psicológicos relacionados à ansiedade, levando-a, por consequência, a pior ajustamento. Esses resultados são semelhantes àqueles relatados em estudos internacionais (Barbaranelli et al., 1998; Caspi et al., 2005; Rothbart \& Bates, 1998; Shiner \& Caspi, 2003).

Acredita-se que o presente estudo apresentou fortes evidências acerca da moderada estabilidade temporal do neuroticismo, corroborando dados internacionais. Dizer que um traço possui estabilidade é assegurar que esse traço sofrerá poucas mudanças decorrentes das influências externas e que essas características acompanharão o desenvolvimento e adaptação do indivíduo ao longo de todo o ciclo vital. Relacionada à estabilidade temporal está a associação encontrada entre o neuroticismo e o funcionamento adaptativo, apontando a influência negativa do traço na adaptação da criança às demandas ambientais. Esse resultado é de suma importância, porquanto alerta para a necessidade da elaboração de estratégias disciplinares e educativas que possam minimizar os efeitos negativos do traço e potencializar os recursos resilientes apresentados pela criança. Os dados são também proveitosos para a Psicologia Escolar e da Educação, uma vez que eles apontam que quanto maior a intensidade do neuroticismo apresentado pela criança pior será seu desempenho na escola. As características de personalidade da criança, além de sua inteligência, devem, pois, ser levadas em consideração quando estratégias de ensino são elaboradas na tentativa de melhorar seu desempenho escolar. No tocante às contribuições para a Pediatria e Medicina, embora o presente estudo não tenha mostrado evidências de associação entre o $\mathrm{N}$ e o IMC, por ser a amostra não clínica, ele sugere que o neuroticismo elevado pode ser um dos determinantes para o desenvolvimento de desordens de cunho nutricional. $\mathrm{O}$ presente estudo aponta, pois, para a necessidade de mais pesquisas que investiguem a relação entre o $\mathrm{N}$ e tais desordens para auxiliar em programas de orientação e na implementação de estratégias de intervenção. O estudo sugere, ainda, que indivíduos mais inteligentes possuem hábitos alimentares e físicos que minimizam a probabilidade de eles desenvolverem problemas nutricionais, como a obesidade e o sobrepeso.

Obviamente, o presente estudo não pretendeu esgotar as possibilidades de relações entre dimensões da personalidade e critérios externos, portanto apontase a necessidade de novas pesquisas em amostras maiores, em faixas etárias diversas e em amostras clínicas com a utilização de outras fontes de informação acerca da personalidade infantil que não somente os autorrelatos.

\section{REFERÊNCIAS}

Achenbach, T. M. (1991). Manual for the CBCL/4-18 and 1991 Profile. Burlington: University of Vermont, Department of Psychiatry.

Barbaranelli, C., Caprara, G., \& Rabasca, A. (1998). BFQ-C Big Five Questionnaire for Children. Firenze: Organizzazioni Speciali.

Batty, G. D., Deary, I. J., Schoon, I. \& Gale, C. R. (2007). Childhood Mental Ability in Relation to Food Intake and Physical Activity in Adulthood: The 1970 British Cohort Study. Pediatrics, 19(1), 38-45.

Caspi, A., Roberts, B. W., \& Shiner, R. L. (2005). Personality Development: Stability and Change. Annual Review of Psychology, 56, 453-484.

Center for Disease Control and Prevention - Departament of Health and Human Services/ Division of Nutrition and Physical Health (2007). Body Mass Index: About BMI for Children and Teens. Recuperado em 30 de janeiro de 2007 em

http://www.cdc.gov/nccdphp/dnpa/bmi/childrens_BMI/abo ut_childrens_BMI.htm

Centro Editor de Psicologia Aplicada - CEPA (2001). Manual das Matrizes Progressivas de Raven - Escala Geral. Séries $A, B, C, D e E$. Tradução e adaptação: Francisco Campos. Rio de Janeiro: CEPA. $2^{\mathrm{a}}$ ed.

Chamorro-Premuzic, T. \& Furnham, A. (2004). A possible model for understanding the personality-intelligence interface. British Journal of Psychology, 95, 249-264. 
Chamorro-Premuzic, T., Furnham, A. \& Petrides, K. (2006). Personality and Intelligence - The relationship of Eysenck's Giant Three with Verbal and Numerical Ability. Journal of Individual Differences, 27(3), 147-150.

Chamorro-Premuzic, T., Furnham, A., Dissou, G. \& Heaven, P. (2005). Personality and preference for academic assessment: A study with Australian University students. Learning and Individual Differences, 15(4), 247-256.

Cournot, M., Marquié, J. C., Ansiau, D., Martinaud, C., Fonds, H., Ferrières, J., Fesc, M. S \& Ruidavets, J. B. (2006). Relation between body mass index and cognitive function in healthy middle-aged men and women. Neurology, 67, $1208-1214$

Csabi, G., Tenyi, T. \& Molnar, D. (2000). Depressive symptoms among obese children. Journal of Eating and Weight Disorders, 5(1), 43-45.

De Raad, B. (2000). The Big Five Personality Factors - The Psycholexical Approach to Personality. Seattle: Hogrefe \& Huber Publishers.

Eysenck, H. J. \& Eysenck, S.G. (1998). Manual of the Eysenck Personality Questionnaire (Junior \& Adult). Adaptado por Nicolas Seisdedos e Agustín Cordero. Madrid: TEA Ediciones. (Original publicado em 1975).

Eysenck, H. J. (1959). Estudio cientifico de la personalid (E. Loedel, trans.). Buenos Aires: Editorial Paidos. (Original publicado em 1952)

Faith, M. S., Flint, J., Fairburn, C. G., Goodwin, G. M. \& Allison, D. B. (2001). Gender Differences in the Relationship between Personality Dimensions and Relative Body Weight. Obesity Research, 9(10), 647- 650.

Jensen, A.R. (1998). The g factor. London: Praeger.

Kovacs, Y. \& Plomim, R. (2006). Generalist genes: implications for the cognitive sciences. Trends in Cognitive Sciences, 10(5), 198-203.

Laidra, K., Pullmann, H. \& Allik, J. (2007). Personality and intelligence as predictor of academic achievement: A crosssectional study from elementary to secondary school. Personality and Individual Differences, 42(3), 441-451.

Mansur-Alves, M. (2007). Diferenças individuais da dimensão de neuroticismo em população escolar por meio dos testes $B F Q-C$ e EPQ-J. Dissertação de mestrado não-publicada, Faculdade de Filosofia e Ciencias Humanas,Universidade Federal de Minas Gerais. Belo Horizonte, MG.

Matthews, G., Deary, I. J. \& Whiteman, M. C. (2003). Personality Traits. London: Cambridge University Press. $2^{\mathrm{a}}$ ed.
Nederkoorn, C., Braet, C., Van Eijs, W., Tanghe, A. \& Cansen, A. (2006). Why obese children cannot resist the food? The role of impulsivity. Eating Behaviors, 7(4), 315-322.

Neisser, U., Boodoo, G., Bouchard, T. J., Boykin, A. W., Brody, N., Ceci, S. J., Halpern, D. F., Loehlin, J. C., Perloff, R., Stenberg, R. J., \& Urbina, S. (1996). Intelligence: Knows and Unknowns. American Psychologist, 51(2), 77-101.

Pacheco, L. M. B. (2003). Comportamento de escolares: aspectos acadêmicos e psicossociais na sala de aula. Tese de Doutorado, Faculdade de Educação, Universidade Estadual de Campinas. Campinas, SP.

Roberts, B. W. \& DelVecchio, W. F. (2000). The rank-order consistency of personality traits from childhood to old age: A quantitative review of longitudinal studies. Psychological Bulletin, 126, 3-25.

Rothbart, M. K. \& Bates, J. E. (1998). Temperament. Em: W.Damon \& N.Eisenberg (Eds.), Handbook of Child Psychology: Vol. 3. (5 ${ }^{\text {th }}$ ed., pp.105-176). New York: Wiley.

Rubinstein, G. (2006). The big five and self-esteem among over-weight dieting and non-dieting women. Eating Behaviors, 7(4),355-361.

Shiner, R. L. \& Caspi, A. (2003). Personality differences in childhood and adolescence: measurement, development, and consequences. Journal of Child Psychology and Psychiatry, 44(1), 2-32.

Shiner, R. L. (1998). How Shall We Speak of Children's Personalities in Middle Childhood? A Preliminary Taxonomy. Psychological Bulletin, 124(3), 308-332.

Stein, L. M. (1994). TDE - teste de desempenho escolar: manual para aplicação e interpretação. São Paulo: Casa do Psicólogo.

Terracciano, A., Costa, P. T. Jr. \& McCrae, R. R. (2006). Personality Plasticity After Age 30. Personality and Social Psychology Bulletin, 32(8), 999-1009.

World Health Organization- WHO. (2006). Health Topics: Obesity and Overweight. Recuperado em 30 de janeiro de 2007 em http://www.who.int/topics/obesity/en/

Recebido em 23/09/2008 Aceito em 27/11/2008

Endereço para correspondência : Marcela Mansur-Alves. Laboratório de Avaliação das Diferenças Individuais/UFMG. Av. Antonio Carlos, 6627, sala 4046, Campus Pampulha, CEP 31270-901, Belo Horizonte-MG, Brasil. E-mail: marcelamansuralves@yahoo.com.br 